\title{
Más allá del autor. La construcción pública del diseño de indumentaria en Argentina
}

\section{Miguel, Paula}

Resumen:

Este trabajo analiza una dimensión particular en la producción del diseño de indumentaria en Argentina: la difusión del término "diseño de autor", a partir del año 2000 , en un recorrido que muestra la difusión pública de un concepto mientras que el diseño de indumentaria se valoriza y consolida como espacio de producción. Se profundiza sobre el rol de la prensa como instancia de mediación que introduce novedades a sus lectores y construye interpretaciones. Por último, se trabaja sobre la perspectiva de los diseñadores y las tensiones que emergen a propósito de los usos del término desde lógicas diferentes.

Palabras clave: Producción simbólica diseño - indumentaria - moda - prensa - "diseño de autor".

(*) Socióloga (UBA), especializada en sociología de la cultura y análisis cultural. Doctora en Ciencias Sociales (UBA). Profesora e investigadora de la Universidad de Buenos Aires.

Introducción

Este trabajo se propone analizar una dimensión particular en la conformación del espacio de producción del diseño de indumentaria1 en la ciudad de Buenos Aires a partir del año 2000. Bajo la premisa de que la producción de los objetos culturales abarca no sólo su producción material, sino también su producción simbólica, este artículo se ocupa de desentramar la difusión pública del término "diseño de autor" en un recorrido que permita comprender el proceso de valorización, legitimación y creciente autonomía que adquiere el diseño de indumentaria desde el año 2000 en adelante. Estos procesos involucran la acción de un conjunto de diferentes actores e instituciones (revistas, museos, espacios académicos, periodistas, diseñadores, entre otros), 
que a través de sus intervenciones y discursos participan de la producción simbólica de los productos culturales (Bourdieu 1990, 1995, 2003).

Particularmente, aquí interesa resaltar aquellas dimensiones que tienen que ver con procesos de intermediación simbólica más amplios que permiten interpretar en públicos amplios el agregado de diseño en los productos como "valor". En ese sentido, si en los inicios de los años 90 era muy raro que se escuchara hablar de diseño de indumentaria y hacia el año 2005 se puede encontrar una categoría nativa como "diseño de autor" que se instala sólidamente en el público para dar cuenta de productores y productos específicos; significa que estamos ante diferentes procesos de intermediación que trabajan activamente en la construcción y difusión de categorías explicativas, al tiempo que contribuyen a la valoración de todo el espacio y proceso productivo.

El artículo propone revisar algunos indicadores de la difusión del término, particularmente en la prensa escrita especializada en moda a partir del año 2000. Esa producción escrita tiene un rol importante en la vinculación inicial con públicos amplios y la forma en que el diseño profesional fue puesto en circulación en diferentes espacios y poco a poco fue reconocido y valorado como un espacio de producción específico. A continuación se profundiza sobre ese rol de la prensa como instancia de mediación que introduce novedades a sus lectores, retomando elementos que contextualizan esos emergentes y construyen interpretaciones sobre los mismos. Por último, se trabaja sobre la perspectiva de los dise- ñadores y las tensiones que emergen a propósito del término.

\section{Estrategia de abordaje}

El acceso a las prácticas, representaciones y la perspectiva de los actores se trabaja a partir del análisis de sus discursos, abordándolos a partir de la situación social en la que se producen. Esto implica tener en cuenta y analizar las posiciones de los actores en el espacio del cual forman parte, así como las redes que construyen. Para eso, este análisis recupera y combina diferentes fuentes, datos y estrategias de abordaje, ya que el uso complementario de distintas estrategias, contribuye a reducir el riesgo de sesgos y limitaciones. Esto permite resaltar, pero de manera crítica y balanceada, la perspectiva de los actores en un espacio particular analizado de manera relacional (Bourdieu, Passeron, y Chamboredón 2002; Sautu 2003; Guber 2004).

Un conjunto de datos cualitativos surge a partir de la construcción de una base de notas de prensa a partir del análisis de los suplementos de moda y revistas dominicales de los principales diarios argentinos2, revistas de moda y artículos recopilados desde 1993 en adelante. Mediante el procesamiento de los textos que proveen los artículos es posible plantear un contexto de uso de distintos términos que se han difundido por medio de la prensa escrita y sus ecos en otros medios de comunicación. Estos datos se complementan con una serie de frecuencias de uso de términos específicos en páginas y sitios en internet. Un segundo conjunto de datos proviene de una línea de investigación en curso centrada el análisis de los productores y emprendedores de las industrias creativas en Buenos Aires3, que recopila sesenta entrevistas en profundidad, para el perfil profesional, con preguntas acerca de las trayectorias profesionales, las condiciones económicas, su experiencia social y cultural, recuperando los hitos vitales que, en las percepciones de los entrevistados, marcan las decisiones relevantes en relación con la actividad profesional4 . También se realizaron observaciones en el contexto de las entrevistas, así como en espacios y eventos representativos para el sector, a lo cual se suman los datos provenientes de muchas otras conversaciones informales y no estructuradas. 
En los primeros años de la década de 2000, comienza a circular el término "de autor", asociado a con la emergencia de nuevos productores en las industrias creativas de la ciudad de Buenos Aires desarrollan sus emprendimientos en el contexto de la crisis de 2001 en Argentina. Podría decirse que, así como los años 80 tuvieron lo "moderno" como síntesis de elementos novedosos en la circulación de productos y productores culturales y artísticos; y los noventa se abrieron a lo "alternativo" y lo "independiente"; los primeros años del siglo veintiuno incorporarían el elemento "de autor". Mientras que "alternativo" o "moderno" podía remitir a una actitud más bien general (Urresti, 1997, pp. 235-236); los términos “independiente" y "de autor" tienen que ver más estrictamente con espacios y formas de producción específica, por ejemplo en cine, editoriales, compañías de teatro, sellos discográficos, gastronomía y diseño, entre otros.

La categoría "diseño de autor", asociada a la indumentaria, comenzó a circular justamente para dar cuenta de un emergente novedoso en la producción. Poco a poco, esa categoría se instaló en el uso cotidiano para dar cuenta de todo un conjunto de producciones en indumentaria, textiles y accesorios que no podían ser atribuidas a las marcas locales ya instaladas o a los modistos más tradicionales y que mayormente se asocian a emprendimientos centrados en la producción de diseñadores formados en la universidad, particularmente la Universidad de Buenos Aires5 . La actividad de los diseñadores profesionales comenzó a desarrollarse a mediados de los años 90, cuando las primeras cohortes de estudiantes egresan de la mencionada carrera, desarrollan emprendimientos comerciales que comienzan a afianzarse en los primeros años dos mil. A partir de las interpretaciones de periodistas de moda y especialistas vinculados al diseño profesional, se agrupó bajo este concepto a los diseñadores profesionales que se pusieron al frente de sus emprendimientos, el estilo de diseño que producían y los espacios donde se los podía encontrar (Miguel, 2013).

Para ilustrar la difusión del término en un contexto de uso amplio, es posible cuantificar la cantidad de resultados para búsquedas sobre la frase exacta "diseño de autor", dentro de páginas de internet. Esos resultados reflejan la frecuencia de registro de la frase dentro de diferentes formatos de publicación, tales como noticias, entradas de blogs, sitios de marcas, entre otras. Dicha búsqueda arroja un promedio de casi 2.500 coincidencias por año registradas entre 2001 y 2005, que trepan a 22.000 resultados en 2010 y más de 100.000 en 2015 (Google Advanced Search, 2017)6. Al desagregar esos resultados de búsquedas por país, según muestra el Gráfico 1, se aprecia la manera en la cual el término "diseño de autor" tiene una presencia más temprana y más cuantiosa en Argentina, en comparación otros países de la región como Chile, Perú y Uruguay, particularmente a partir de 2007, haciendo referencia muchas veces al caso argentino como antecedente específico (Calvo Foxley, 2013).

Esos resultados de búsqueda simplemente muestran un comportamiento general en la frecuencia de uso de un término en la web, sin embargo, no aportan datos sobre el contexto de uso. Para reponer la forma en que se difunde el término, se trabaja a continuación sobre los artículos publicados en la prensa argentina especializada en moda, que cumple un rol importante en el dar a conocer el diseño profesional y contribuir a difundir la actividad de productores y sus productos.

La difusión del término en la prensa: primeras menciones

En la prensa local casi no se hacen referencias al diseño de indumentaria con anterioridad al año 2000, si bien podría haber datos disponibles desde 1994-1996, cuando los primeros graduados de la carrera de diseño de 
indumentaria de la Universidad de Buenos Aires, por ejemplo, comenzaban a hacer visibles sus producciones. Poco a poco comenzó a difundirse el diseño de indumentaria, vinculado a las carreras universitarias y las producciones de sus egresados.

En esas primeras referencias se destacan rasgos que proponen ese diseño como emergente de culturas juveniles que buscan diferenciarse, que se profesionalizan, pero al mismo tiempo tienen un elemento "creador" distintivo:

En el circuito de diseñadores jóvenes, se escucha decir que en la Argentina la gente usa uniformes. De allí la importancia de una nueva generación que estudia diseño de moda y que tiene la ilusión del creador, que no copia lo que se hace en el exterior, sino que diseña con talento y creatividad, según sus propios cánones (La Nación, 4 de abril de 1999).

Alrededor de los años 2001 y 2002, la categoría "diseño de autor" comenzó a expresar la síntesis de un conjunto de producciones que no podían ser atribuidos a las marcas comerciales locales, o a los modistos tradicionales, sino que se asociaron con los diseñadores egresados de carreras universitarias, fundamentalmente de la Universidad de Buenos Aires:

De unos pocos años a esta parte, la carrera de Diseño de Indumentaria hizo explotar un circuito inédito: decenas de nuevos nombres se suman al mundo de la moda, aportando una visión lúdica al hecho de vestirse. Ahora, merced a políticas nacionales y porteñas, Buenos Aires intenta convertirse en el nuevo polo de diseño del Mercosur (Página/12, 27 de abril de 2001).

Aunque en muchos casos aparecen elementos diversos y difusos para definir este tipo de diseño, se evidencia un esfuerzo por aludir a una producción específica que logró atravesar los peores años de la crisis económica, destacando su potencial en términos de negocio:

El diseño de autor argentino, en general, se caracteriza por los cortes elaborados, costuras a contratono, apliques, avíos de colores, detalles de alforzas y bordados, morfologías nuevas. (...) La devaluación posibilitó una inserción del diseño argentino en el exterior porque llegó a ser, con sus precios, competitivo (La Nación, 12 de diciembre de 2002).

El término "diseño de autor" registra a medida que pasa el tiempo una mayor frecuencia de uso, se perfila como un segmento diferenciado y se van delimitando cada vez más claramente espacios asociados a este tipo específico de diseño (Miguel 2009, 2015). La zona de Palermo en la ciudad de Buenos Aires, por ejemplo, se va definiendo como "EL" barrio de diseño: "Considerado el Soho porteño, Palermo ofrece mucha ropa y accesorios de autor, de acuerdo con la tendencia a personalizar la moda" (La Nación, 4 de agosto de 2001).

Paralelamente, la semana de la moda llamada BAF Week gana presencia en las crónicas de la prensa como "EL" evento donde se condensó la producción de los diseñadores profesionales y sus emprendimientos. Ya para el año 2005, muchas de las crónicas sobre esa semana de la moda apuntaban como el principal protagonista del evento el "diseño de autor", no "la moda" local ni algún otro término más genérico o alguna marca o diseñador en particular: "Cinco años de un encuentro que se consolida como la principal pasarela de diseño de autor" (La Nación, 6 de marzo de 2005). 
De esta manera, se delimitan zonas y eventos específicos que expresan una forma de conocimiento espacializado sobre la moda (Entwistle, 2010) y permiten circunscribir la energía colectiva, movilizada por un conjunto de actores, que se manifiesta de manera amplificada y concentrada durante esos eventos que ocurren en momentos determinados (Entwistle y Rocamora, 2006). Poniendo en relación personas, eventos, zonas geográficas, espacios de formación, diseño y diseñadores de indumentaria, productores y productos, va condensándose un significado para el término "diseño de autor". El término recuperó en una síntesis, elementos diversos como experiencias artísticas, el quehacer de jóvenes profesionales, la creatividad o la innovación, así como la coyuntura de un momento histórico específico, tal como la crisis de 2001 en Argentina. El apartado siguiente explora las formas en que se realizó esa construcción.

\section{La construcción de un concepto}

A partir de las referencias visibilizadas en las notas de prensa surge la pregunta a propósito de cómo se conforman esas construcciones de significado. ¿Cómo se articula la relación diseñadores y público / revista y lectores? ¿Qué elementos permiten introducir elementos novedosos? ¿Cómo se articularon elementos diversos para conformar la categoría "diseño de autor"?

La prensa y la crítica especializada en moda tienen un rol importante en la difusión de novedades vestimentarias vinculando a la producción con el público. A lo largo de la década de 1970, en Argentina, como en muchos otros países, aparecieron cada vez más revistas sobre moda dirigidas mayoritariamente a un público femenino. Esa producción escrita tuvo un carácter fuertemente propositivo y las publicaciones acompañaban las dinámicas de circulación de la moda en su divulgación "traduciendo" las tendencias emergentes al público. Hacia los años 90, el público se volvió más complejo y fragmentado, recortado según estilos de vida y ocupación o intereses de sus lectores; por otro, cobraron mayor visibilidad los estilistas o diseñadores como "creadores" de colecciones, donde apareció cada vez con más fuerza la firma como parte del producto. Ambos fenómenos contribuyeron a modular la forma en que se comunica desde la prensa (Todeschini Borges, 2006).

En Argentina, las revistas con producción de contenido local, que pueden ser de tradición nacional (como Para $\mathrm{Ti}$, creada en 1922) o bien, franquicias de revistas internacionales, junto con los suplementos de moda de los diarios más importantes, dedican sus contenidos a las lectoras, que son en cierta medida potenciales consumidoras; no se trata de una prensa que produzca para el sector industrial, sino que más bien se apunta a un universo de lectoras que cada revista define o imagina a priori (Bontempo, 2006; Cosse, 2011). El esfuerzo editorial por definir estilos y explicar las tendencias estéticas emergentes se orientaba hacia esos segmentos específicos del universo de lectoras y se manifestó en la construcción de un discurso específico que las editoras trabajaban entre sus lectoras "ideales" y sus lectoras "reales", a las cuales deben interpelar:

Primero partes de la identidad que le quieres dar a tu revista, a tu sección y ahí, una vez que tienes definido eso, tienes la otra punta que es tu lectora. Yo a esa mujer le tengo que llegar (...) Hay un conocimiento de la lectora, de tu producto, sabes lo que sí y sabes lo que no, sabes hasta dónde puedes tirar de la piola y cuando no. Te encuentras con las dificultades de la necesidad de definir, de poner palabras, de explicar (Entrevista a editora de Revista Para Ti, 2002).

Esa forma de intervención tiene que ver con una lógica particular en la cual la moda vestimentaria deviene relato, pasa a ser un objeto cultural relativamente autónomo, con una finalidad que se ajusta a la lógica de la 
comunicación escrita, donde constituye un sistema propio, una estructura que permite en una construcción de sentido, clasificar y distribuir en una jerarquía los objetos vestimentarios (Barthes, 1967, pp. 307-312). No obstante, esa "moda escrita" (Rocamora, 2009, pp. 54-62) no solamente describe o valora objetos en abstracto, en un sistema cerrado, sino que también los construye. El discurso sobre la moda influye en el proceso de producción de los bienes de moda y su valoración (Bourdieu y Delsaut, 1975). Es decir, la prensa tiene como prioridad sostener el interés en su revista, que se piensa como producto. Debe mantener la atención de sus lectores, que muchas veces se definen como target a partir de investigaciones de mercado. Debe incorporar y comunicar novedades, lo cual contribuye a construir definiciones, valoraciones y jerarquías en un sistema más amplio donde convergen lógicas múltiples, dando lugar a tensiones que emergen en ese solapamiento. Al mismo tiempo se construye un sistema de clasificación ritualizado que instituye géneros diferenciados que se organizan jerárquicamente (Di Maggio, 1987). En ese sentido, el “diseño de autor” se construyó paulatinamente, incorporando novedades en un universo de lectores que debían ser definidos o explicados. En el desarrollo de esa dimensión explicativa se recurrió a distintos elementos que permitieron ubicar esa innovación en un contexto, en una serie, en el marco de tradiciones preexistentes que marcan una continuidad histórica respecto de antecedentes (Blumer, 1969).

La "moda escrita" fue exponiendo y construyendo un nexo entre los diseñadores y el mundo del arte, elaborando un relato que puede ser leído como la construcción de una genealogía, de un pasado para ese "diseño de autor" en Argentina. Para ilustrar ese fenómeno se seleccionan dos trabajos de periodistas argentinas (Lescano, 2004 y Acevedo Díaz, 2011), editados como libros que de alguna manera constituyen un registro más formal, en tanto se constituyen como volúmenes de referencia, más allá de las notas periodísticas eventuales. Lescano (2004), por ejemplo, recupera tres momentos en el desarrollo de la moda local. Los años sesenta y setenta están representados por la vanguardia artística del Instituto Di Tella en los años sesenta, donde algunos artistas pivotaban entre la producción de vestimenta y la experimentación artística, como por ejemplo, Dalila Puzzovio, Mary Tapia y Rosa Bailón quien tenía su boutique "Madame Frou Frou" ubicada en una galería cercana a dicho instituto (King, 2007 y Saulquin, 2006).

En un segundo momento, se presentó la indumentaria vinculada con la producción artística de las generaciones más jóvenes, los fashion happenings de fines de los años 80 y la Primer Bienal de Arte Joven, realizada en 1989. Gabriel Grippo, Kelo Romero y Sergio de Loof fueron los personajes emblemáticos de esta fase productiva que se vinculaba también con otros espacios y personajes del under, como vestuaristas de obras de teatro del circuito off o de bandas de rock, organizadores de fiestas y discotecas, entre otros7. De esta manera, el arte de los jóvenes y ciertas estéticas de vanguardia son el hilo conductor en esa mirada, conformando un pasado para los "autores" que representaban la moda de los años 90 y 2000; un grupo conformado mayormente por diseñadores profesionales.

El trabajo de Acevedo Díaz (2011, p. 15) también describe el fenómeno "diseño de autor" como gestado a fines de los años 80, que adquirió mayor visibilidad desde fines de los noventa y que se transformó en suceso a partir del año 2001, donde tienen un papel relevante los egresados de distintas carreras de diseño, ente otras. En sintonía con esos trabajos periodísticos, también es posible encontrar trabajos analíticos que coinciden en la perspectiva que vincula el "diseño" con el momento de efervescencia en la producción estética y artística característica de la vuelta a la democracia en los años 80, la creación de la carrera de Diseño de Indumentaria y Textil en la Universidad de Buenos Aires, y la crisis de 2001 como el momento en que esas producciones cobran visibilidad (Saulquin, 2006, 2011; Tuozzo y López, 2013). 
Susana Saulquin, una referente en el análisis del sistema de la moda local, reflexionó tempranamente sobre estos fenómenos. Señala que:

Un diseño es considerado de autor cuando el diseñador resuelve necesidades a partir de su propio estilo e inspiración, sin seguir las tendencias que se imponen desde los centros productores de moda (...) con una concepción basada en la personalidad y en la comunicación de cierta identidad. (...) Además, revalorizan la actividad artesanal que desconoce las series industriales y ponen al alcance de las personas modas múltiples que descreen de mandatos únicos (Saulquin, 2006, p. 16).

Más tarde, la categoría "diseño de autor" permea algunas instancias de diagnóstico sobre el sector de la indumentaria. En esa línea, la Fundación ProTejer, que reúne a distintos actores de la cadena de valor del textil y la indumentaria, junto con el Instituto Nacional de Tecnología Industrial (INTI), realizaron en el año 2010 la primera Encuesta Nacional de Diseño de Indumentaria de Autor en Argentina (Marino et al. 2010). Allí se define:

Entran en la categoría diseño de indumentaria de autor aquellas empresas que diseñan y producen prendas con valor agregado a partir de procesos de trabajo intensivos que incorporan alta dosis de creatividad y experimentación en uno o más aspectos del producto, como ser la morfología y/o textura y/o simbología de las piezas. (...) el criterio de originalidad se evidencia a través de productos que logran escapar a las tendencias de moda masivas, a través de lenguajes creativos innovadores sostenidos en el tiempo (Marino et al. 2010, p. 13).

Otros análisis posteriores recuperan el uso del término en línea con esa definición que propone la mencionada encuesta (Mon, 2012, 2013; Chiesa et al. 2012; Veneziani, 2013), junto a otros trabajos académicos que también recuperan el uso del término (Galán, 2008; Correa, 2011; Joly, 2012; Doria, 2014 y Marré, 2014, entre otros). Algunos enfoques tienden a trabajar el concepto como categoría nativa, es decir, resaltando su pertenencia a un contexto de uso específico, en tanto producto de prácticas específicas, que no necesariamente funciona en el análisis como categoría explicativa en sí misma o con un sentido unívoco (Guerschman y Vargas, 2008; Miguel, 2009, 2013; Vargas, 2013 y Zambrini, 2016, entre otros).

Estas distintas fuentes permiten dar cuenta de un término extendido en el uso para dar cuenta de la forma de pensar en un estilo específico de diseño, que circula en ciertos espacios, primero de manera más restringida, luego amplificándose en el uso (Douglas, 1998) pero que, no obstante, presenta algunas aristas problemáticas que se hacen notar a medida que pasa el tiempo y se modifican contextos y coyunturas.

La mirada de los productores

¿De qué manera es posible medir o cuantificar "altas dosis de creatividad" o de "inspiración"? ¿Qué observables se ofrecen para su análisis? Sin lugar a dudas, el término presenta cuestiones que constituyen un desafío a ser resuelto en un análisis relacional que piense la producción simbólica como construcción colectiva.

Partiendo de la premisa de que la producción de diseño se ubica en tensión entre dos lógicas: la de la producción artística/estética y aquella concerniente a la producción material/económica8 , a medida que nos separamos de la producción más genérica y se pone el foco en los espacios de producción más exclusivos en el mundo de la moda, encontramos similitudes u homologías entre la alta costura o alto diseño con la lógica del mundo artístico (Bourdieu y Delsaut, 1975; Bourdieu, 1990). En ese plano, se presentan una serie de relaciones 
en torno a la convicción sobre el valor de una obra o los diseños en este caso, mediante procedimientos propios de una lógica carismática que presenta un mundo de "creadores", "creativos" naturalmente dotados por el don de la "inspiración"; un mundo donde los intermediarios son también "descubridores inspirados", apasionados por las obras, con un don especial y/o natural que les hace percibir su valor intrínseco y que deniega en esa operación de consagración los elementos económicos que están en juego. En el marco de esa creencia es eficaz una ideología carismática que origina la cuestión del productor aparente, del creador increado: la figura del genio, el iluminado, el creador, el autor y la creencia en esos roles (Bourdieu, 1995; 2003). En ese sentido, la lógica del "autor" tiende a escindir de las prácticas la dimensión material y económica, mediante procedimientos opacos, lo cual presenta tensiones a medida que la creencia en ese valor pierde efectividad.

Ese encuentro de diferentes lógicas permite explicar cómo lo que hace sentido para los intermediarios, como los periodistas de moda, puede presentarse de manera diferente entre los diseñadores. Los diseñadores muchas veces no encuentran en el "diseño de autor" una categoría que los exprese, o que los defina, incluso entre aquellos diseñadores que fueron parte de los primeros egresados en la carrera de diseño, que fueron los primeros en establecerse en el barrio de Palermo y que actualmente se catalogan como "consagrados" por la crítica, se registran posiciones que buscan diferenciarse tanto de las experiencias artísticas previas a la profesionalización y la formación de carreras de diseño de indumentaria en el ámbito universitario, como de lo que posteriormente sedimenta como "diseño de autor".

Para ilustrar estas tensiones se han seleccionado cinco casos ilustrados a partir de los testimonios de diseñadores-emprendedores. Estos testimonios abarcan casos que han formado parte del grupo de "pioneros" de este fenómeno, cuyos emprendimientos se desarrollaron alrededor del año 2000 (entrevistas A, B y C) junto a trayectorias más recientes de emprendimientos gestados después de 2010 (entrevistas D y E)9

En relación con los antecedentes vinculados a la producción más artística, al analizar las percepciones de los diseñadores, se evidencia que, en muchos casos, sus percepciones no coinciden unívocamente autoreferenciándose en dichos antecedentes. Por sobre la impronta artística se destaca un elemento clave que es lo que hace a un diseñador: la formación profesional:

Eran gente que no tenía formación universitaria. Tenemos como inicios diferentes. Respeto eso que se generó en ese momento pero siento que tuve un origen distinto. (...) Yo realmente ya tengo una formación que es como más rigurosa, casi matemática. Por más que yo me dé las licencias de que en mi proyecto yo pongo el sentir ante todo, mi estructura mental tiene sus parámetros, formas, organización, sé cómo se arma producción, como se arma una serie... es distinto (Entrevista A - Diseñadora-Empresaria, 2005).

Los diseñadores que se desarrollaron profesionalmente al frente de sus propios emprendimientos en los temprano años 2000, se piensan ante todo como un grupo profesional. En ese sentido, su quehacer es diferente de producciones a las que se le reconocen interés y se valoran en distintos niveles pero que, pertenecen a otro espacio, a otra lógica, propio de las manifestaciones artísticas que tienen que ver con gestos de vanguardia, que se interpretan como locos o inviables, desde el concepto de diseño aplicado a un emprendimiento comercial. Estos diseñadores tuvieron en muchos casos una formación centrada en el estudio del diseño como área proyectual, junto con una valoración del área académica en el marco de carreras universitarias, lo cual también se observa en otras áreas cercanas como diseño gráfico o industrial (Fernández y Bonsiepe, 2008; Devalle, 2009). A propósito del término "diseño de autor", los diseñadores resaltan de manera crítica que esa concepción 
oculta de alguna manera la dimensión que tiene que ver con el desarrollo de los emprendimientos en sentido comercial o económico (McRobbie, 1998), como por ejemplo, la construcción de marca. Por otra parte, el “diseño de autor" tiende a asociarse a producciones equiparables a la de un artista: 'DDiseño de autor' me parece que es como elevar las cosas que haces a una cosa más artística. Esto es ropa para transpirar, abrochar, desabrochar, agacharse, caminar, meterla en el lavarropas, subirte a un colectivo" (Entrevista C - DiseñadoraEmpresaria, 2016). O bien, a producciones demasiado experimentales o extravagantes: "A veces, se encasilla el diseño de autor en ropa rarísima y re poco ponible" (Entrevista D - Diseñadora-Empresaria, 2015).

El remitir al autor aparece, en la perspectiva de estos diseñadores, como oposición a la funcionalidad del diseño, que se piensa con un objetivo y un usuario. La figura del autor, al mismo tiempo, tiene el efecto de disociar el diseño de su producción material:

Al autor lo veo más encerrado en su atelier y haciendo un vestido rarísimo. Lo que hacemos nosotros es industrial, no somos artesanales, todo lo que se hace es industrial y se produce como se produce en cualquier marca, como si fuera producto masivo. Siempre entendimos que nosotros hacemos ropa y no creo eso de que nosotros, como diseñadores, seamos artistas (Entrevista B - Diseñador-Empresario, 2016).

Esa separación entre autor e industria, por otra parte, opaca el trabajo colectivo presente en la producción y que se piensa como una tarea que articula el trabajo de muchas personas, al tiempo que se rescata la intención de construir identidad de marca más que identidad de autor, con un sentido de construcción simbólica potente que apunta a un fin comercial:

A mí, la nomenclatura de 'diseño de autor' no es algo que me guste. Me parece que sí, somos autores, pero está el equipo que también aporta. Nuestra idea es manejar esto como se maneja una marca pero con diseño, pensando, siendo originales pero, como se maneja una marca (Entrevista B - DiseñadorEmpresario, 2016).

Los diseñadores detectan términos que se "imponen" desde fuera. En el caso de la tensión entre marca y autor, esta se percibe como algo propio de la escena amplia del diseño de indumentaria local, donde -desde una posición ajena a la de los diseñadores-emprendedores-, se proponen como opuestos elementos que para ellos no lo son. Muy a menudo se resalta el hecho de buscar constituirse en un plano que resuelva esa tensión, proponiéndose como una marca con diseño: "Es rara toda esa división. Acá o sos diseñador o sos una marca. En el mundo, sos diseñador y tenés una marca, es lo mismo. Nosotras nos pensamos como una marca con diseño" (Entrevista D - Diseñadora-Empresaria, 2015). Desde la lógica del diseñador-emprendedor, el desafío es crecer como marca, poder desarrollar no sólo el concepto o la línea proyectual de diseño individual, sino también todo aquello que permitirá comercializarlo. Construirse como marca, no opaca el rol del dise- ñador sino más bien lo contrario, de hecho muchos de los emprendimientos de diseño catalogados y consagrados como "de autor" realizan alianzas con distintas firmas que les permiten producir y comercializar en volúmenes mayores, ganar visibilidad en un público más amplio.

Por otra parte, sobre todo entre los emprendimientos más jóvenes, se identifica el elemento "de autor" como una fórmula que se aplica sin diferenciación a un sinnúmero de casos y que, de tanto repetirse, ha perdido su significado: “También impusieron eso del nombre de 'diseño de autor', que ya lo podríamos superar todos y dejar de usarlo porque no sé ni qué significa. ¿Qué es el diseño de autor? Es rarísimo. ¿De autor de qué?” (Entrevista D - Diseñadora-Empresaria, 2015). 
Se plantea el desafío sobré cómo "seguir manteniendo un carácter innovador y bohemio". Propuestas, que en un momento era únicas, diversas y diferenciadas, pueden llegar a percibirse como un continuum indiferenciado de copias de una fórmula ganadora que se reitera hasta el hartazgo: "Hay también un abuso del término 'de autor'. Todo es 'de autor' ahora... planta de autor, sillón de autor. Es como que se encapsulan cosas y queda rarísimo. Ya no se sabe ni de qué hablamos" (Entrevista E - Diseñadora-Empresaria, 2016). Esta situación que se identifica como repetición en el plano discursivo tiene además un correlato territorial, por ejemplo en las percepciones sobre el barrio de Palermo (Miguel, 2015). El efecto de repetición e indiferenciación presenta dos aspectos problemáticos: por un lado, la oferta diferenciada a la cual hacía referencia el término "diseño de autor" en sus orígenes se entremezcla con nuevas propuestas estandarizadas en el marco de su propia clave estética. Por otro, aparece la cuestión sobre la repetición de la fórmula "de autor" que enfrenta la proliferación de infinidad de productores y productos que saturan la posibilidad de experimentar algo "distinto y único". En los últimos años comienzan a reflejarse ecos de estas tensiones en las notas de prensa que recogen testimonios de diseñadores:

En el circuito local el diseño de autor se montó en una imposición y se vació de contenido, razón por la cual necesita resignificarse. $Y$ en eso estoy desde mi firma, en la búsqueda de una resignificación, después de trece años en el mercado (Entrevista M. D., Página/12, 31 de mayo de 2013).

En ese sentido, el término "diseño de autor", colocado en el centro de referencia de la construcción pública sobre el diseño de indumentaria, encuentra limitaciones para sostener su centralidad a medida que es posible encontrar una oferta consolidada y diversificada de producción local de diseño de indumentaria que ha crecido en distintos segmentos de mercado y escalas productivas.

\section{Comentarios finales}

Los procesos vinculados a las dinámicas de producción simbólica van más allá de la consolidación de espacios académicos, curriculares o profesionales. En el caso del diseño de indumentaria en Argentina, se presentan particularidades: pese a estar inscripto en un mercado global, mantiene características locales; pese a estar orientado al mercado y a formas de consumo cada vez más estetizados, rige en algunas zonas de su producción la lógica del "talento" o de la "creación", entre otras tensiones que es posible explorar.

Un "diseño de autor" sólo es un objeto simbólico provisto de valor si es conocido y reconocido como tal, o, en otras palabras, si está instituido socialmente por un conjunto de productores y públicos que detentan las competencias necesarias para conocerlo y reconocerlo en esos términos. Bajo esta perspectiva, comprender este tipo de producciones implica analizar no sólo la producción material, sino también la producción del valor simbólico, como creencia en ese valor (Bourdieu, 1995, p. 339) y, no menos importante, la creencia en el valor de sus productores, y de aquellos que pueden ser capaces de apreciar "genuinamente" el valor de esa obra que es, en última instancia, una empresa colectiva.

Así, una cuestión a resolver en los análisis sobre la producción artística, estética y cultural tiene que ver con cómo se producen y reproducen estos productores y sus obras. Para eso, resulta necesario ubicar en un plano relacional la producción y circulación de bienes culturales, poniendo el foco tanto en los productores, como en las instancias de intermediación, intermediarios y un público cercano, comprometido, que en cierta medida comparten una creencia, en el valor de esos bienes. Esa es una tarea colectiva, que requiere la movilización de 
energía y recursos compartidos, en distintas proporciones, por el conjunto de agentes implicados en el campo. El valor de la obra dado por la creencia surge, en última instancia, del conjunto de las prácticas que se dan entre productores, consumidores y quienes hacen a la mediación entre ellos de diversas maneras.

Retomando esa línea, en el caso del diseño de indumentaria en Argentina, la prensa especializada, tuvo un rol importante para la difusión en un público amplio de un término que diera cuenta del diseño profesional que emerge en los primeros años dos mil. Al mismo tiempo, hacia el interior del espacio de producción de diseño, la prensa contribuyó en el trabajo de consagración, construyendo referentes, proponiendo nuevos "autores", dando definiciones que permiten organizar un espacio jerárquico de posiciones y al mismo tiempo transmitirlas. Esa tarea clasificatoria, permite construir una trayectoria en términos simbólicos, es decir una reputación, un "nombre".

Al mismo tiempo, estas instancias, que generan la ilusión de ser formas de juicio o valoración mayormente estéticos, tienen efectos económicos en la medida en que esas definiciones guían y orientan las tendencias entre compradores y vendedores, contribuyen a conformar el valor económico de los productos. Analizar el proceso de difusión pública de términos como "diseño de autor" resulta productivo para comprender procesos de valoración simbólica y cómo dicha producción se articula con la producción material y económica sobre la base de la intervención de un conjunto de personas e intermediaciones, donde convergen diferentes lógicas y elementos económicos, sociales y culturales más amplios.

El término "diseño de autor" es parte de un proceso de objetivación y sedimentación que es necesario revisar en tanto categoría analítica. Los conceptos en uso no constituyen representaciones estáticas sino más bien construcciones y modos performáticos de construir visiones sobre el mundo. En esa línea, este trabajo propone entender el contexto de emergencia del término, su evolución en el tiempo como elemento dinámico, productivamente diferenciador y clasificador, así como las relaciones entre actores vinculados con su producción y reproducción, teniendo en cuenta las tensiones que emergen en esa convergencia colectiva. Esa perspectiva abre la posibilidad de repensar y evaluar la productividad de estas categorías para dar cuenta más o menos acabada de los fenómenos a los cuales remiten en distintos momentos y contextos productivos. Al mismo tiempo, plantea la necesidad de pensar el diseño más allá del autor, poner en suspenso las definiciones que emergen de las perspectivas o lógicas particulares e indagar sobre sus condiciones de producción para incorporar contextos y categorías que repongan miradas complejas y relacionales en el análisis.

\section{Notas}

1. En las últimas décadas en Argentina, particularmente en la ciudad de Buenos Aires, se evidenció un desarrollo significativo de las llamadas industrias creativas, que abarcó distintas áreas de producción de bienes y servicios. Particularmente, el diseño aplicado a la producción de indumentaria y textiles experimentó un crecimiento destacado en la ciudad de Buenos Aires. Entre 2004 y 2010 la actividad de confección de prendas de vestir, terminación y teñido de pieles, considerada industria de diseño intensiva, experimentó una variación del 176.26\% (Observatorio de Industrias Creativas 2012, p. 88).

2. Los diarios Clarín, La Nación, Página/12 publican contenidos que permiten condensar enunciados e interpretar distintos elementos en relación con la moda en un público más amplio que el de las revistas de moda, aunque muestren producciones más simples que las revistas sobre moda. 
3. El trabajo de campo se realizó en el marco de los proyectos UBACYT 20020110200278BA y

20020130200279BA, acreditados y financiados por la Universidad de Buenos Aires.

4. Las entrevistas surgen de una muestra según propósitos o selección basada en criterios; no es estática sino que refiere a un proceso en curso, con diseño abierto (Maxwell, 1996, p. 6). La selección de informantes responde al ajuste de los intereses de investigación o necesidades emergentes (Holstein y Gubrium, 1995, p. 74).

5. Un exponente muy dinámico del diseño profesional se vincula con la carrera de Diseño de Indumentaria y Textil (DIT) en la Facultad de Arquitectura y Urbanismo de la Universidad de Buenos Aires. Esta carrera se creó en los años 1988-1989 y representó un paso importante hacia la institucionalización y profesionalización del diseño en esas áreas de producción en Argentina. Ha sido y continúa siendo de las más numerosas del país en matrícula de estudiantes y logró posicionarse como referente en la región, ganando un reconocimiento internacional gracias a los trabajos y logros de sus estudiantes, egresados y docentes.

6. Si bien la penetración del uso de Internet se acrecienta a lo largo de los primeros años dos mil, muchas publicaciones de la región ofrecen sus archivos para consulta de noticias publicadas anteriormente, desde los años noventa, lo cual permite rastrear la presencia del término en la prensa con anterioridad al uso extendido de Internet.

7. Sobre estos antecedentes y cruces entre diferentes espacios de producción artística, puede consultarse el corpus de entrevistas presentado en la compilación de Lucena y Laboureau (2016).

8. Un uso heurístico de las líneas que Pierre Bourdieu propone para el análisis del campo intelectual y artístico $(1990,1995,2000,2003)$ resultan productivas para comprender y explicar estos fenómenos.

9. Agradezco especialmente la colaboración de Lucía Aizemberg Tolosa en la realización del trabajo de campo realizado entre 2015 y 2016.

Bibliografía

Acosta, A. et al. (2013). INTI Mapa de diseño 101 diseñadores de autor. San Martín: INTI.

Barthes, R. (1967). Système de la mode. Paris: Éditions du Seuil.

Bergamo, A. (2007). A experiência do status: roupa e moda na trama social. San Pablo: UNESP.

Bontempo, P. (2006). Para Ti: Una revista moderna para una mujer moderna (1922-1935), Estudios Sociales, 41: 127-156.

Bourdieu, P. (1990). Alta costura y alta cultura. En: Sociología y cultura. México: Grijalbo. (1995). Las reglas del arte: génesis y estructura del campo literario. Barcelona: Anagrama. (2000). La distinción: criterios y bases sociales del gusto. Madrid: Taurus. 

(2003). Creencia artística y bienes simbólicos: elementos para una sociología de la cultura.

Argentina: Aurelia Rivera.

Bourdieu, P.; Passeron, J. C. y Chamboredón, J. C. (2002). El oficio del sociólogo. Presupuestos epistemológicos. Buenos Aires: Siglo XXI.

Bourdieu, P. y Delsaut, Y. (1975). "Le couturier et sa griffe : contribution à une théorie de la magie." Actes de la recherche en sciences sociales 1:7-36.

Calvo Foxley, S. (2013). Relatos de moda. Santiago de Chile: Salviat Impresores.

Correa, M. E. (2011). La producción cultural del diseño. El caso de los diseñadores independientes de la ciudad de Buenos Aires. Trabajo y sociedad, (17), 329-342.

Cosse, I. (2011). Claudia: la revista de la mujer moderna en la Argentina de los años sesenta (1957-1973). Mora (Buenos Aires), 17(1).

Devalle, V. (2009). La travesía de la forma. Emergencia y consolidación del Diseño Gráfico (1948-1984). Buenos Aires: Paidós.

Dimaggio, P. (1987). Classification in art. American Sociological Review, 52, (4), 440-455.

Doria, P. (2014). Sobre la Enseñanza del Diseño de Indumentaria: El desafío creativo (enseñanza del método). Cuadernos del Centro de Estudios en Diseño y Comunicación. Ensayos, (48), 37-47.

Douglas, M. (1998). Estilos de pensar. Ensayos críticos sobre el buen gusto. Barcelona: Gedisa.

Entwistle, J. y Rocamora, A. (2006). "The field of fashion materialized: a study of London Fashion Week. Sociology 40 (4), 735-751.

Fernández, S. y Bonsiepe, G. (Comp.) (2008). Historia del diseño en América Latina y el Caribe. Sao Paulo: Editora Blücher.

Galán, M. B. (2008). Diseño y complejidad en la cátedra de Metodología de la Carrera de Diseño Industrial. Trabajo práctico de diseño estratégico con materiales del Delta del Paraná. Huellas, Búsquedas en Artes y Diseño, 6:22-39.

Guber, R. (2004 [1991]). El salvaje metropolitano. Reconstrucción del conocimiento social en el trabajo de campo. Buenos Aires: Paidós.

Guerschman, B. y Vargas, P. (2007). Quilombo y apuesta: apuntes etnográficos sobre la crisis argentina de 2001 a través de la mirada del mundo del diseño. Avá, (11), 39-62.

Holstein, J. y Gubrium, J. (1995). The active interview. Thousand Oaks: Sage Publications. 
Joly, V. (2012). Arte, moda y consumo cultural. Cuadernos del Centro de Estudios en Diseño y Comunicación. Ensayos, (39), 283-294.

Lucena, D. y Laboureau, G. (comps.) (2016). Modo mata moda. Arte, cuerpo y (micro) política en los ‘80. La Plata: EDULP.

Marino, P. et al. (2010). Diseño de indumentaria de autor en Argentina. Buenos Aires: INTI.

Marré, S. (2014). El asociativismo en las empresas de diseño de indumentaria de autor en Argentina. Cuadernos del Centro de Estudios en Diseño y Comunicación. Ensayos, (48), 59-69.

Maxwell, J. A. (1996). Qualitative research design: An interactive approach. London: Sage Publications.

Miguel, P. (2009). Los recorridos del diseño de indumentaria en la ciudad de Buenos Aires. Apuntes de Investigación del CECyP, 15:47-69.

Miguel, P. (2013). Emprendedores del diseño. Aportes para una sociología de la moda. Buenos Aires: Eudeba.

Miguel, P. (2015). La estetización del espacio y la espacialización de lo estético en Buenos Aires. Una mirada desde la producción. Bifurcaciones. Revista de Estudios Culturales Urbanos, 20:1-16.

Mon, L. (2012). Industrias Creativas de Diseño de Indumentaria de Autor. Diagnóstico y desafíos a 10 años del surgimiento del fenómeno en Argentina. Cuadernos del Centro de Estudios en Diseño y Comunicación 42:19:34.

Mon, L. (2013). Diseño en Argentina: Hacia la construcción de nuevos paradigmas. Cuadernos del Centro de Estudios en Diseño y Comunicación. Ensayos, (44), 83-99.

McRobbie, A. (1998). British fashion design. Rag trade or image industry. Londres: Routledge.

Observatorio de Industrias Creativas (2012). Anuario de Industrias Creativas Ciudad de Buenos Aires 2011. Buenos Aires: Ministerio de Desarrollo Económico, GCBA.

Pérez Zelaschi, M. (2012). Observatorio de Tendencias. Cuadernos del Centro de Estudios en Diseño y Comunicación. Ensayos, (42), 35-42.

Rocamora, A. (2006). Paris Capitale de la Mode: Representing Fashion in the Media. In: Fashion's World Cities. Berg Publishers, pp. 43-54.

Rocamora, A. (2015). High Fashion and Pop Fashion: The Symbolic Production of Fashion in Le Monde and The Guardian. Fashion Theory (5),2: 123-142.

Sautu, R. (2003). Todo es teoría. Objetivos y métodos de investigación. Buenos Aires: Lumiere.

Stivale, S., \& González Trigo, M. (2012). El mercado de productos de diseño sustentables en la ciudad de Mar del Plata, situación actual y expectativa. InvestigacióN + AccióN, (14), 139 -160. 
Todeschini Borges, V. (2006). Uma questão de estilo: a cobertura de moda na mídia impressa carioca. Rio de Janeiro: Prefeitura, Secr. Especial de Comunicação Social.

Tuozzo, M. V. y López, P. (2013). Moda y Arte. Campos en intersección. Cuadernos del Centro de Estudios en Diseño y Comunicación. Ensayos, (44), 123-134.

Urresti, M. (1997). Los modernos: una nueva bohemia posvanguardista. En: Mario Margulis (et al.), La cultura de la noche: la vida nocturna de los jóvenes en Buenos Aires. Buenos Aires: Biblos.

Vargas, P. (2013). Diseñadores y emprendedores. Una etnografía sobre la producción y el consumo de diseño en Buenos Aires. La Plata: Al Margen.

Veneziani, M. (2013). Moda y Arte en el diseño de autor argentino. Cuadernos del Centro de Estudios en Diseño y Comunicación. Ensayos, (44), 71-82.

Veneziani, M. (2014). El enfoque multidisciplinario: un desafío pedagógico en la Enseñanza de la Moda y el Diseño. Cuadernos del Centro de Estudios en Diseño y Comunicación. Ensayos, (48), 19-22.

Zambrini, L. (2016). De diseñadoras, diseñadores y diseños. Reflexiones desde una perspectiva de género. Iconofacto. (11), 100-110.

Artículos periodísticos

"Que sepa coser...", La Nación Revista, 4 de abril de 1999.

“La nueva ola”, Página/12, 27 de abril de 2001.

“En moda, el diseño personal encuentra un nuevo espacio”, La Nación, 4 de agosto de 2001.

“Denim toda la vida”, La Nación, 12 de diciembre de 2002.

“En busca de la identidad”, Página/12, 31 de mayo de 2013 (entrevista a DI Mariana Dappiano).

“Jazmín Chebar. ‘Nunca entendí qué es el diseño de autor”, La Nación, 30 de mayo de 2015 (entrevista a DI Jazmín Chebar).

Abstract: This paper analyzes a particular dimension in the production of clothing design in Argentina: the dissemination of the term "signature design", starting in 2000. The article showcases the public diffusion of a concept while fashion design is valorized and consolidates as a space of production. It explores on the role of the press as an instance of mediation that introduces novelties to its readers and constructs interpretations. Finally, it presents the perspective of designers and the tensions that emerge about the uses of the term from different logics.

Key words: Symbolic production - design - clothing - fashion - press - "signature design". 
Resumo: Este trabalho analisa uma dimensão particular na produção de design de indumentária na Argentina: a difusão do termo "design de autor", a partir do ano 2000, num percurso que mostra a disseminação pública de um conceito enquanto o design de roupa se valoriza e consolida como espaço de produção. $\mathrm{O}$ artigo aprofunda sobre o papel da imprensa como uma instância de mediação que apresenta novidades aos seus leitores e constrói interpretações. Finalmente, o trabalho se foca na perspectiva dos designers e as tensões que emergem em torno aos usos do termo a partir de diferentes lógicas.

Palavras chave: produção simbólica - design - indumentária - moda - imprensa - "design de autor".

[Las traducciones de los abstracts fueron supervisadas por el autor de cada artículo]

Más allá del autor. La construcción pública del diseño de indumentaria en Argentina fue publicado de la página 161 a página177 en Cuadernos del Centro de Estudios de Diseño y Comunicación N76 